\title{
Cloning, expression and activity of ATP-binding protein in Bacillus thuringiensis toxicity modulation against Aedes aegypti
}

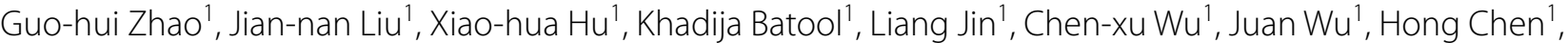 \\ Xiao-yan Jiang ${ }^{1}$, Zhao-hui Yang ${ }^{1}$, Xian-hui Huang ${ }^{1}$, En-jiong Huang ${ }^{2}$, Xiao-Qiang Y Y ${ }^{3,4}$, Xiong Guan ${ }^{1}$ \\ and Ling-ling Zhang ${ }^{1 *}$
}

\begin{abstract}
Background: Bacillus thuringiensis israelensis (Bti) is a widely used mosquitocidal microbial pesticide due to its high toxicity. ATP-binding proteins (ABP) are prevalently detected in insects and are related to reaction against Bti toxins. However, the function of ABP in mosquito biocontrol is little known, especially in Aedes aegypti. Therefore, this study aimed to clarify the function of $A B P$ in Ae. aegypti against Bti toxin.

Results: Aedes aegypti ABP (GenBank:XM_001661856.2) was cloned, expressed and purified in this study. Far-western blotting and ELISA were also carried out to confirm the interaction between ABP and Cry $11 \mathrm{Aa}$. A bioassay of Cry $11 \mathrm{Aa}$ was performed both in the presence and absence of $A B P$, which showed that the mortality of Ae. aegypti is increased with an increase in ABP.

Conclusions: Our results suggest that ABP in Ae. aegypti can modulate the toxicity of Cry 11 Aa toxin to mosquitoes by binding to Bti toxin. This could not only enrich the mechanism of Bt toxin, but also provide more data for the biocontrol of this transmission vector.
\end{abstract}

Keywords: Aedes aegypti, ATP-binding protein, Biocontrol, Bti, Interaction

\section{Background}

Mosquitoes, such as Anopheles, Aedes and Culex, are important infectious medium in transmitting various tropical diseases. Global health problems associated with mosquito-borne diseases put hundreds of millions of people at risk [1-3]. For example, Aedes spp. are the causative agent for dengue hemorrhagic fever and Zika virus $[4,5]$. The attempt to control and early detection of these infections becomes a global public health issue [4]. However, there are no valid vaccines or medicines available presently. Vector control is the main method for preventing the spread of these diseases $[2,6,7]$.

*Correspondence: lingling00264@163.com

1 State Key Laboratory of Ecological Pest Control for Fujian and Taiwan Crops \& School of Life Science, Fujian Agriculture and Forestry University, Fuzhou 350002, China

Full list of author information is available at the end of the article
Presently, mosquitoes are mostly controlled by chemical pesticides. However, traditional chemical pesticides can cause environmental pollution and human health problems. Furthermore, it is easy for insect resistance to develop [8]. Nowadays, biological pesticides, in particular microbiological pesticides, are increasingly promoted due to being more environmentally friendly and having higher specificity, less influence on non-target organisms and inducing less resistance. Hence, microbiocontrol is regarded as one of the best means of control, and the application of entomopathogens to control mosquito populations is particularly effective [9].

Bacillus thuringiensis (Bt) is a Gram-positive bacterium used worldwide in biological control due to its high specificity to insects and low impact on the environment [10]. It can produce some pore forming toxins (PFTs, including Cry toxin and Cyt toxin) in its sporulation phase, which play an important role in the process of killing insects $[11$, 
12]. They are toxic to more than 3000 species of insect in different orders, including Lepidoptera, Coleoptera and Diptera, etc. Bacillus thuringiensis israelensis (Bti) is widely used for its high toxicity against mosquitoes. It can produce a composite mosquitocidal crystal protein containing Cry2, Cry4, Cry10, Cry11, Cyt1 and Cty2 [1315]. Among them, Cry11Aa can be activated by hydrolysis with proteinase in the mosquito midgut, and displays high toxicity to larvae of Aedes and Culex, but low toxicity against Anopheles gambiae [9, 15]. Once mosquito larvae have been exposed to the toxin, the activated toxin can bind to specific receptors to form an oligomer in the brush border membrane vesicles (BBMV) of the midgut [16-18], including the alkaline phosphatase (ALP), aminopeptidase N (APN), cadherin and ATP-binding cassette $(\mathrm{ABC})$ transporters [9]. Oligomerization of toxins and insertion of toxin oligomers into the midgut epithelial cells cause perforation and cell death $[16,18]$. However, some details in this process are still unclear, especially about the interaction of toxin and receptor [19]. Recently, the resistance of Bt toxin, which can diminish the toxicity in many insects, has drawn the public eye. As reported with organophosphate insecticides, some third-part proteins besides toxins and receptors can also be related to the insect resistance [8]. Because of the similar binding site, galectin could make Caenorhabditis elegans resistant to Cry5Ba toxin by binding to the receptors such as lipids and glycolipids [20]. In our previous work, we found that galectin-14 of Aedes aegypti can compete with Cry11Aa by binding to some of the Cry receptors, such as ALP1 [21]. Galectin- 6 was also found to interact with ALP1 to affect the toxicity of Cry (unpublished results). C-type lectin can help Anopheles gambiae prevent Plasmodium infection via humoral immunity [22]. C-type lectin-20 was also found to interact with ALP1 to reduce Cry toxicity in Ae. aegypti [23]. Resistance can also be induced by the mutation of ATP-binding cassette $(\mathrm{ABC})$ transporter in Heliothis virescens [24-28].

$\mathrm{ABP}$ is a large group of proteins that can hydrolyze ATP to provide energy for the transmembrane transport process. Recently, ABP was reported to play a role in the pathology of insects reacting to Bt toxin. A single amino acid mutation in $\mathrm{ABP}$ was found to improve the resistance of Bombyx mori against Bt toxin [29]. After an amino acid was inserted in ABP C2, the modified gene was expressed in the Sf9 cell. Bioassays showed that the modified ABP was less sensitive to Cry1A toxin in $B$. mori than the control ABP without modification [30]. After ABP G was silenced by RNAi, Plutella xylostella was found to be less sensible to the Cry toxin. As peroxidase $\mathrm{C}$ was found to be a Cry1 Ab-binding protein in Spodoptera exigua [19], ABP in Cx. quinquefasciatus was also found to change the toxicity of Cry11Aa by interacting with this toxin [31]. These results all show that $\mathrm{ABP}$ might relate to the mechanism of Cry toxin against insects.

However, the function of $\mathrm{ABP}$ in mosquito biocontrol is still unclear, especially in Ae. aegypti. The aim of the present study was to clarify the function of $\mathrm{ABP}$ in $A e$. aegypti against Bti toxin.

\section{Methods \\ Mosquitoes, bacterial strains, antibodies and plasmids}

Aedes aegypti Haikou strain was supplied by the Fujian International Travel HealthCare Center and maintained in our laboratory in an environment-controlled room at approximately $28{ }^{\circ} \mathrm{C}$ and $85 \% \mathrm{RH}$ with a photoperiod of $14 \mathrm{~h}$ light and $10 \mathrm{~h}$ dark. The Cry11Aa recombinant Bt strain was kindly provided by Dr Sarjeet Gill, University of California, Riverside, CA, USA. Escherichia coli JM109 and BL21 were preserved in the Key Laboratory of Ecological Pest Control for Fujian and Taiwan Crops, Fujian Agriculture and Forestry University, and cultivated in Luria-Bertani (LB) liquid medium (10 g/l tryptone, $5 \mathrm{~g} / \mathrm{l}$ yeast extract, $10 \mathrm{~g} / \mathrm{l} \mathrm{NaCl}, \mathrm{pH}$ 7.2). Bt LLP29 was isolated and preserved in our previous work [32]. The rabbit polyclonal antibody against biotin, goat-anti-rabbit-APconjugated polyclonal antibody and the streptavidin horse-radish peroxidase (HRP) conjugate were purchased from Beyotime (Shanghai, China). The polyclonal antibody against Cry11Aa was produced in rabbits using purified recombinant Cry11Aa as an antigen [31]. The pMD18-T and pET-32a plasmids were purchased from TaKaRa (Dalian, China) and Novogene (Beijing, China), respectively.

\section{Acquisition of the ABP gene}

Total RNA was extracted from Ae. aegypti using an E.Z.N.A. ${ }^{\circledR}$ Total RNA Kit I (Omega Norcross, GA, USA), then reverse-transcribed using a PrimeScript ${ }^{\circledR} 1$ stStrand cDNA Synthesis Kit (TaKaRa, Dalian, China), both according to the respective manufacturer's instructions. The ABP gene (XM_001661856.2) was amplified by polymerase chain reaction (PCR) with specific primers designed according to the gene sequence in the National Center for Biotechnology Information (NCBI) database as follows: ATPF: 5'-GGA ATT CCA TAT GCA AAA TAA AGT GGT AAC CCT CAA AAC G-3'(NdeI restriction site underlined), ATPR: 5'-ACG CGT CGA CGC TCA CAT ACG GAT TGA TGT CCC GTT-3' (SalI restriction site underlined). NdeI/SalI enzyme sites were selected for restriction digestion. The PCR product was purified using a DNA Gel Extraction Kit (Omega), and ABP gene was sequenced by Sangon Biotech (Shanghai, China). 


\section{Cloning, expression and purification of $\mathrm{ABP}$}

In order to clone the ABP gene in Ae. aegypti, the purified PCR product was ligated with pMD-18T and then transformed into E. coli JM109 as described [31, 33]. Recombinant plasmid was digested with NdeI/SalI and then ligated into the Pet-32 (+) expression vector $[31,33]$. The PCR product of ABP from the recombinant plasmid was also sequenced by Sangon Biotech. The recombinant $\mathrm{ABP}$ was transformed into competent E. coli BL21 cells to express recombinant ABP. A blank Pet-32 (+) plasmid was also transformed into competent E. coli BL21 to express thioredoxin, which was used as a negative control in the subsequent experiments. After being induced by $0.5-1 \mathrm{mM}$ isopropyl- $\beta$ D-1-thiogalactopyranoside (IPTG), the target protein was purified using a Protein ISO Ni-NTA Resin reagent kit (TransGen Biotech,Beijing, China) and then further labeled using EZ-Link-NHS-Biotin (Thermo Fisher Scientific, Waltham, MA, USA), both according the respective manufacturer's instructions. Then, sodium dodecyl sulfate polyacrylamide gel electrophoresis (SDS-PAGE) was carried out to check the recombinant purified ABP [33]. Thioredoxin was also extracted using the same method.

\section{Cry11Aa protein preparation}

The preparation of Cry11Aa protein from the recombinant Bt strain was carried out using the method described by Chen et al. [33-35]. The polyclonal antibodies against Cry11Aa protein were produced in rabbits using purified recombinant Cry11Aa. The secondary antibody goat-anti-rabbit-AP-labeled was detected using a BCIP/NBT alkaline phosphatase assay kit (Beyotime, Nanjing China).

\section{Western blot}

In order to detect the recombinant proteins, western blot was carried out as follows. The biotinylated ABP and purified Cry11Aa were separated by 10\% SDS-PAGE and transferred to a polyvinylidene fluoride (PVDF) membrane, which was further blocked with PBSM (PBS $+0.05 \%$ skim milk) for $2 \mathrm{~h}$ at $37{ }^{\circ} \mathrm{C}$, and washed with PBST (PBS + 0.05\% Tween-20). The membrane was then detected by the primary rabbit polyclonal antibody to biotin for ABP (1:3000) or the primary rabbit polyclonal antibody (1:3000) for Cry11Aa. The goat-anti-rabbit-APconjugated polyclonal antibody (1:3000) was used as the secondary antibody. All antibodies were diluted by PBS with $1 \%$ skim milk. A BCIP/NBT alkaline phosphatase assay kit was used to visualize the result, following the manufacturer's instructions [14, 34, 35].

\section{Far-western blot}

Far-western blot was used to detect the binding characterization between ABP and Cry11Aa protein. The biotinylated ABP and Cry11Aa were separated by $10 \%$ SDS-PAGE and transferred to a PVDF membrane. After being blocked with 5\% dry skim milk in PBS for $2 \mathrm{~h}$ at $37{ }^{\circ} \mathrm{C}$, the membrane with biotinylated $\mathrm{ABP}$ was then probed with the purified Cry11Aa protein $(4 \mathrm{ng} / \mathrm{ml}$, dissolved in PBS (pH 7.4) containing 0.1\% BSA) overnight at $4{ }^{\circ} \mathrm{C}$ with gentle rocking, and then washed three times with PBST. The membrane was then incubated with primary rabbit polyclonal antibody to Cry11Aa (1:3000) and goat-anti-rabbit-AP-conjugated polyclonal antibody (1:3000) was used to detect Cry11Aa. After washing 3 times with PBST and PBS buffer, the binding of the interacting protein was checked by a BCIP/NBT alkaline phosphatase assay kit.

At the same time, the membrane with Cry11Aa was also incubated by biotinylated ABP $(4 \mathrm{ng} / \mathrm{ml})$, following probed by the primary rabbit polyclonal antibody (1:3000) to biotin and the secondary goat-anti-rabbit-APconjugated polyclonal antibody (1:3000). A BCIP/NBT alkaline phosphatase assay kit was used to visualize the binding protein following the manufacturer's instructions [23].

\section{Enzyme-linked immunosorbent assay (ELISA)}

To further understand the interaction between $A B P$ and Cry11Aa, the purified recombinant ABP, Cry11Aa and thioredoxin were biotinylated with EZ-Link-NHS-Biotin following the manufacturer's instructions. ELISA was then carried out as follows. Four micrograms of ABP or Cry11Aa was coated with ELISA buffer $\left(\mathrm{Na}_{3} \mathrm{CO}_{3}, \mathrm{pH}\right.$ 9.6) in 96-well plate overnight at $4{ }^{\circ} \mathrm{C}$, and then washed 3 times with PBS. ELISA buffer was only added to uncoated wells as a negative control. The increased concentration of Cry11Aa, biotinylated ABP or the control biotinylated thioredoxin (0 to $1280 \mathrm{nM}$ ) in $100 \mu \mathrm{l}$ PBST were supplied to the protein-coated wells and further incubated for $2 \mathrm{~h}$ at $37^{\circ} \mathrm{C}$. The plates were then washed 3 times with $100 \mu \mathrm{l}$ of PBST, and the bound biotinylated Cry11Aa, biotinylated ABP or thioredoxin were detected by incubation of the plates with the streptavidin horseradish peroxidase (HRP) conjugate antibody (1:3000) for $2 \mathrm{~h}$. After washing with $100 \mu \mathrm{l}$ of PBST for 3 times, the chromogenic regent kit EL-TMB P0209 (Beyotime BiotechNanjing China) was used for the development of color. Absorbance was checked at $450 \mathrm{~nm}$ on a Multis$\operatorname{kan}^{\mathrm{TM}}$ GO Microplate Spectrophotometer (Thermo Fisher Scientific). Each treatment was repeated 3 times and data were analyzed using GraphPad Prism v.6. 


\section{Bioassays}

For bioassays with the purified ABP and Cry11Aa, thirdinstar Ae. aegypti larvae were fed with purified Cry11Aa protein $(2.0 \mu \mathrm{g} / \mathrm{ml})$ or a mixture of the purified Cry11Aa protein $(2.0 \mu \mathrm{g} / \mathrm{ml})$ and ABP $(0,1.0,2.0,3.0$ or $4.0 \mu \mathrm{g} / \mathrm{ml})$ in $30 \mathrm{ml}$ of dechlorinated water. Thioredoxin was used as the negative control. Each treatment was replicated 3 times. The survival rates of the mosquito larvae were recorded after 12, 24 and $48 \mathrm{~h}$ [36]. The data of bioassays were further analyzed by IBM SPSS Statistics v.19.0.

\section{Results}

\section{Cloning the ABP gene}

The PCR result showed that the full length ABP gene (GenBank: XM_001661856.2) has an open reading frame (ORF) of 915 bp encoding 304 amino acids (Fig. 1). The product was then purified and ligated into the cloning vector $\mathrm{pMD}-18 \mathrm{~T}$ plasmid. After being transformed to JM109, positive colonies were obtained, and the sequence was confirmed by Sangon Biotech. The target fragment was also detected after the recombinant plasmid was digested by NdeI and SalI restriction endonuclease (Fig. 2). The plasmid was then sequenced and the result showed a high homology (up to 99\%) with the initial ATP-binding protein gene. All of these results show

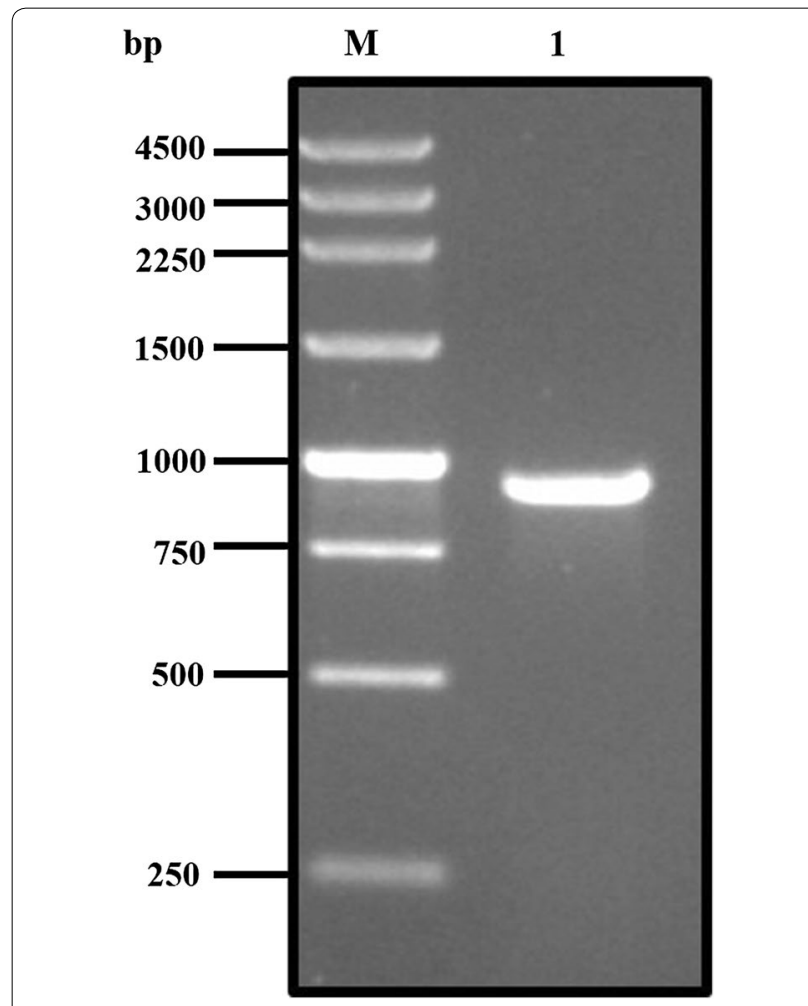

Fig. 1 PCR product of ATP-binding protein. Lane M: 100 bp plus DNA ladder; Lane 1: PCR product of ATP-binding protein

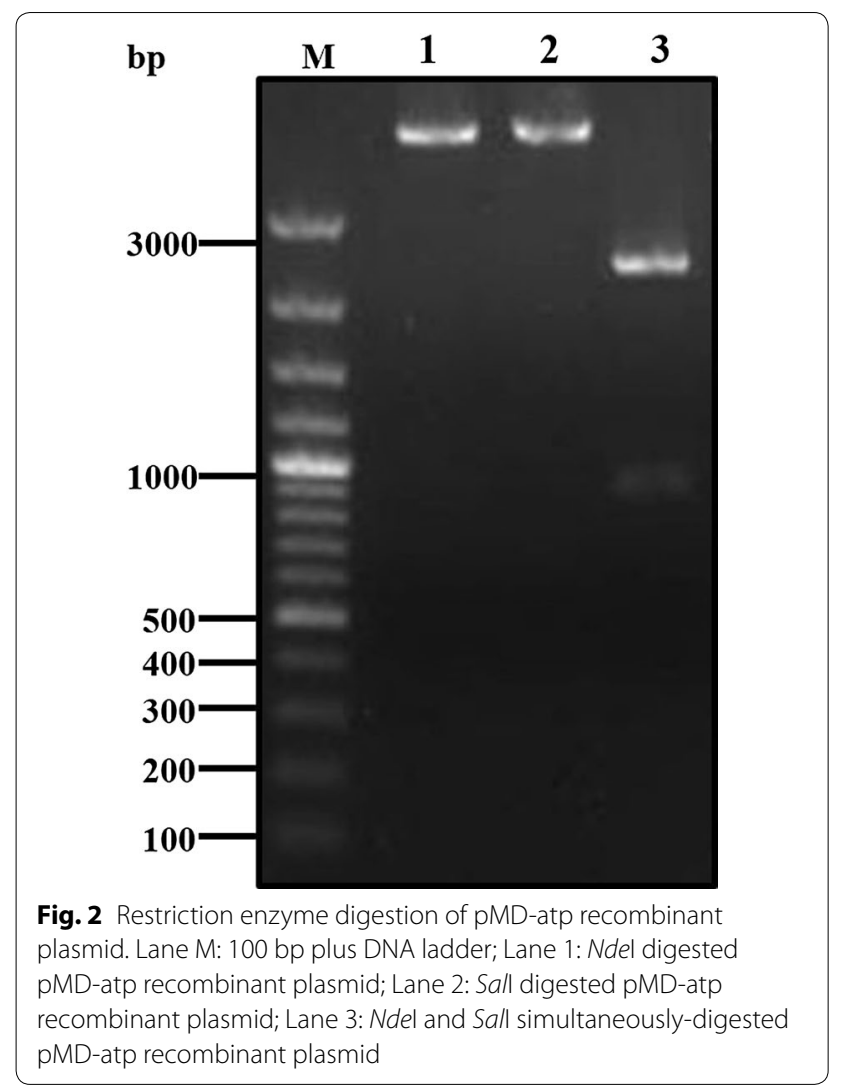

that the gene of the ATP-binding protein was successfully cloned.

\section{Expression and detection of $A B P$ and Cry $11 \mathrm{Aa}$}

Cry11Aa crystal was expressed and purified from the recombined Bt strain that showed a $72 \mathrm{kDa}$ band (Fig. 3a) in SDS-PAGE. Western blot also showed the $72 \mathrm{kDa}$ band detected by the Cry11Aa specific antibody. From this, it can be concluded that Cry11Aa was expressed successfully (Fig. 4a). Meanwhile, the ABP gene was expressed in a prokaryotic expression system by ligating with the PET-32a expression vector. The PET-32a vector plasmid was digested with NdeI and SalI prior to ligation with digested ABP gene from the ABP-pMD-18T recombinant plasmid. After the recombinant plasmid was transformed into E. coli BL21, the positive clones that were confirmed by PCR and enzyme digestion were selected. The sequence was also confirmed by Sangon Biotech. After being induced by $0.5-1 \mathrm{mM}$ IPTG, the expressed ABP was purified using a Protein ISO Ni-NTA Resin reagent kit and labeled with EZ-Link-NHS-Biotin. The result of SDS-PAGE showed a $35 \mathrm{kDa}$ band (Fig. 3b). The western blot also showed that ABP with a $35 \mathrm{kDa}$ band was also detected by rabbit polyclonal antibody against biotin (Fig. 4b). 
$\mathbf{a}$

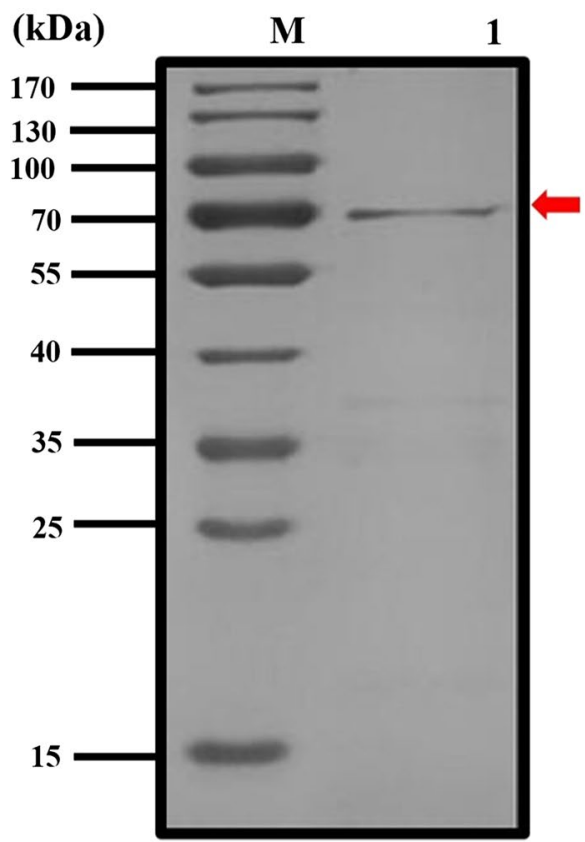

b

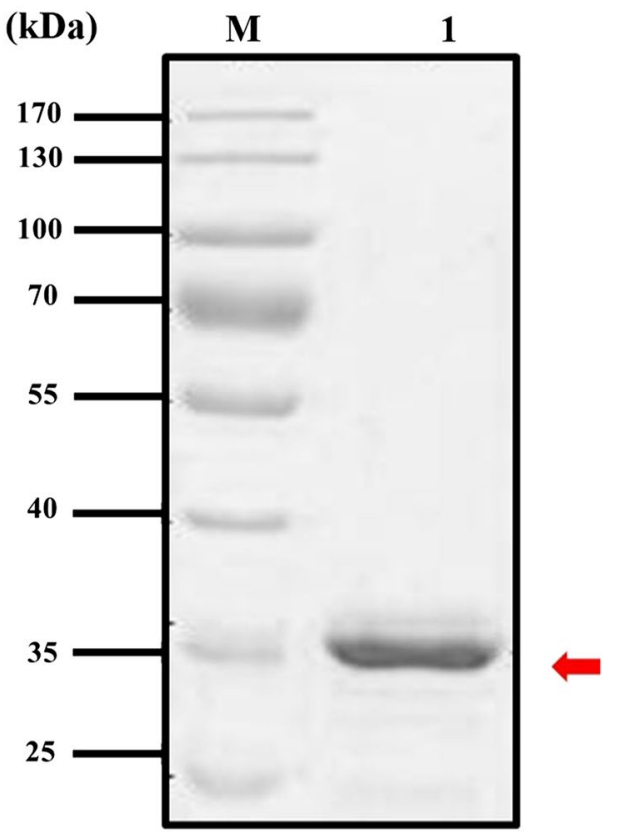

Fig. 3 SDS-PAGE analysis purified Cry11Aa and ATP-binding protein. a SDS-PAGE analysis of the expressed protein (Lane M: protein molecular weight markers; Lane 1: purified Cry11Aa). b SDS-PAGE analysis of the expressed protein (Lane M: protein molecular weight markers; Lane 1: purified ATP-binding protein)

\section{Effect on toxicity of Cry11Aa by ABP}

In order to estimate the function of ABP in Cry11Aa toxicity, a bioassay against Ae. aegypti was carried out. The survival rate against Cry11Aa was recorded after 12, 24 and $48 \mathrm{~h}$ with the presence and absence of ABP. Results showed an obvious drop in the survival rate with increasing concentrations of $\mathrm{ABP}$ (Fig. 5); from this it can be deduced that ABP might enhance the toxicity of Cry11Aa toxin.

\section{Interaction of ABP and Cry11Aa}

To test whether ABP could bind with Cry11Aa, the present study used western blot and far-western blot. After Cry11Aa was separated by SDS-PAGE and incubated with biotinylated ABP, the target band of $72 \mathrm{kDa}$ was detected by specific rabbit polyclonal antibody against biotin (Fig. 6a); no band was detected after Cry11Aa was probed with the negative control thioredoxin. Similarly, when ABP was incubated with Cry11Aa, a band of $35 \mathrm{kDa}$ could be detected by the specific antibody against Cry11Aa (Fig. 6b). These results show that ABP and Cry11Aa could bind to each other.

ELISA was also carried out to further confirm the binding interaction between Cry11Aa and ABP. With an increasing concentration of biotinylated ABP used, more biotinylated ABP was found to bind with the immobilized Cry11Aa. The highest affinity was detected at a concentration of ABP of $1280 \mathrm{nM}$ (Fig. 7a). Similarly, more Cry11Aa also bound to the immobilized ABP with an increased concentration of Cry11Aa and the binding condition was saturated when the concentration of Cry11Aa was up to $640 \mathrm{mM}$ (Fig. 7b). In the negative control, both Cry11Aa and ABP were unable to bind with thioredoxin (Fig. 7). All of these results demonstrate that ABP can interact with Cry11Aa.

\section{Discussion}

ABP is a large group of proteins that can be a transmembrane transporter with the help of ATP hydrolyzation. A significant amount of research shows that ABP plays a vital role in species of Lepidoptera such as Bombyx mori [29, 30], Plutella xylostella [37] and Helicoverpa armigera [38]. It was even found to interact with Cry11Aa and affect the toxicity of Cry11Aa against $C x$. quinquefasciatus in our previous study [33]. However, there is limited research about the function of ABP in Aedes. Therefore, identification of the relationship between ABP of Aedes and Cry11Aa toxin can greatly enrich the knowledge of Bt mechanism.

In the present study, the ABP gene of Ae. aegypti was cloned and expressed to analyze the relationship between $\mathrm{ABP}$ from Ae. aegypti and Cry11Aa toxin from 


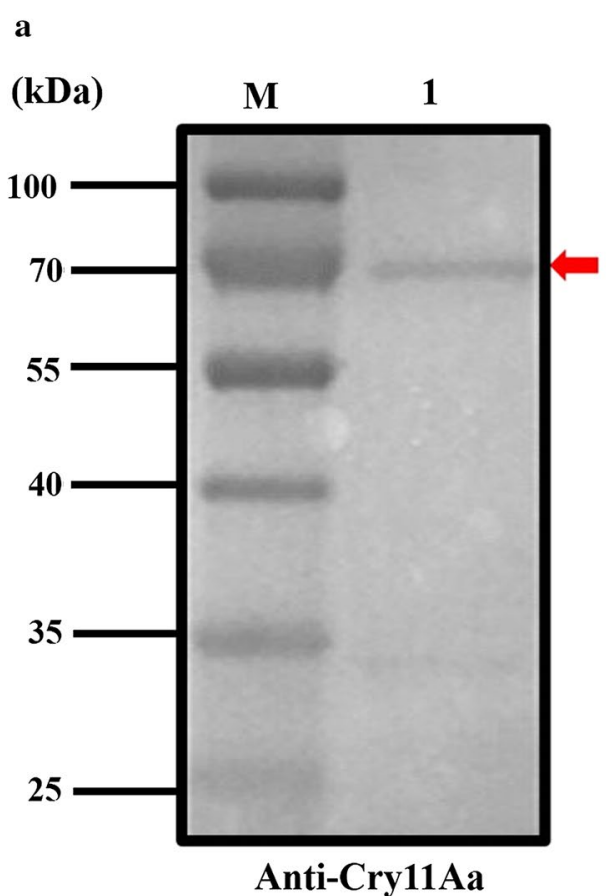

$\mathbf{b}$

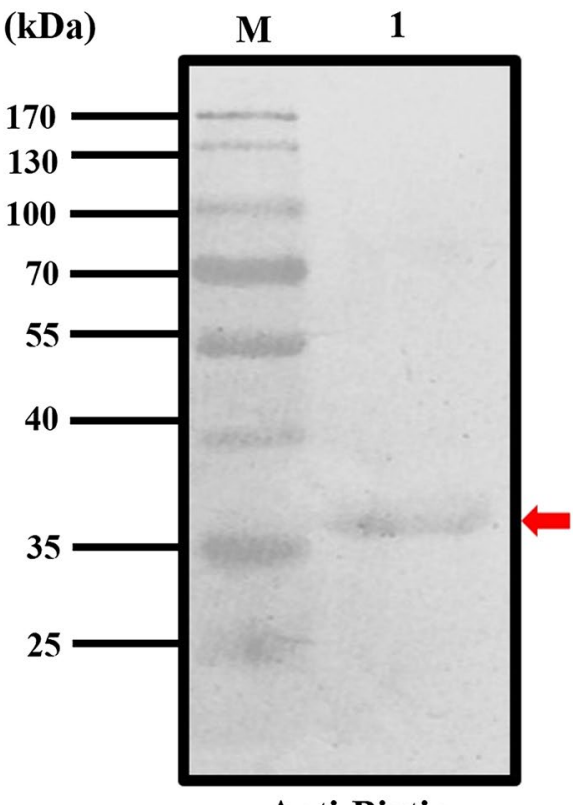

Anti-Biotin

Fig. 4 Western Blot analysis of Cry 11 Aa and ATP-binding protein. a Western blot analysis of the expressed protein (Lane M: protein molecular weight markers; Lane 1: purified Cry11Aa). b Western blot analysis of the expressed protein (Lane M: protein molecular weight markers; Lane 1: purified biotinylated ATP-binding protein)
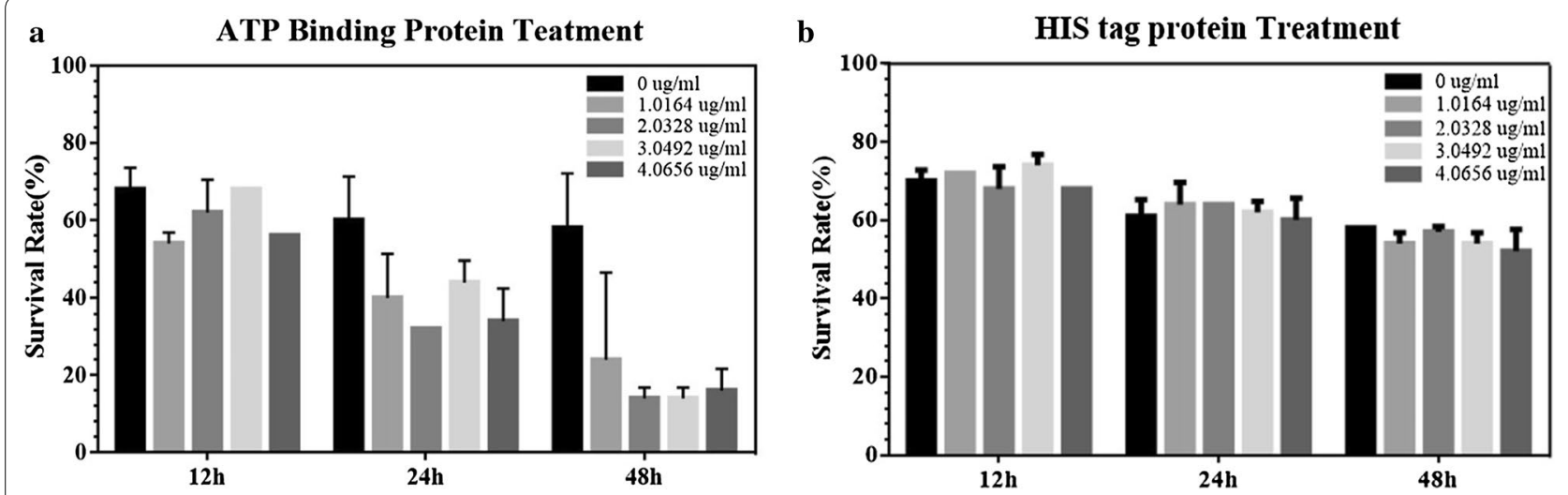

Fig. 5 Bioassay of the ATP-binding protein. Each column represents the mean \pm SEM $(n \geq 3)$. Each bar represents the mean \pm SD of three technical replicates

Bti. Similar to Cx. quinquefasciatus, ABP in Ae. aegypti was found to bind with Cry11Aa toxin. The toxicity of Cry11Aa toxin can be enhanced by ABP through its interaction with this toxin [33]. Although the detailed mechanism of ABP in enhancing the toxicity of Cry11Aa needs further study, our results indicate that ABP can bind effectively to Cry11Aa protein, similar to ALP and APN [9]; the toxicity of Cry11Aa can be influenced by this binding characterization in Ae. aegypti. $\mathrm{ABP}$ may be a factor that can interact with the toxin to form a composite to strengthen the toxicity or influence the physiological process in Ae. aegypti [33]. In order to further understand its function, ABP can be further silenced. Some other proteins in the mosquito midgut could also be tested to see whether they can alter the toxicity of Bt toxins. Such results could not only greatly enrich the knowledge of Bt mechanism, but also contribute to the discovery of new toxin receptors in pests, which could provide more data for the creation of new bio-pesticides. 
a

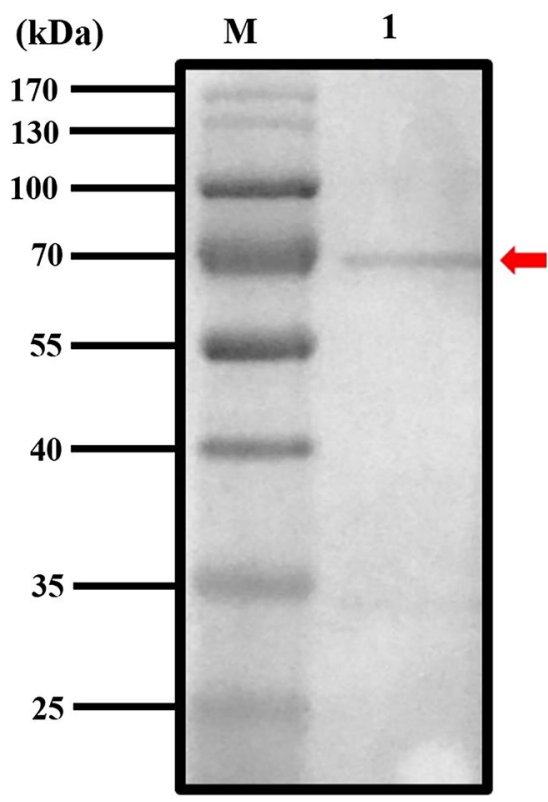

Anti-Biotin b

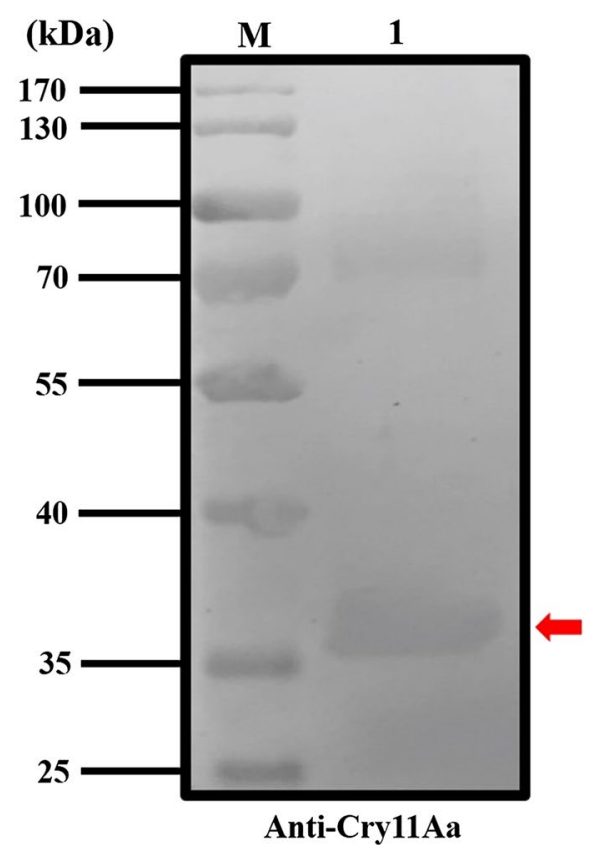

Fig. 6 Far-western blot analysis of Cry 11 Aa and ATP-binding protein. a Far-western blot analysis (Lane M: protein molecular weight markers; Lane 1: purified Cry11Aa probed with biotinylated ATP-binding protein). b Far-western blot analysis (Lane M: protein molecular weight markers; Lane 1: purified ATP-binding protein probed with Cry $11 \mathrm{Aa}$ )
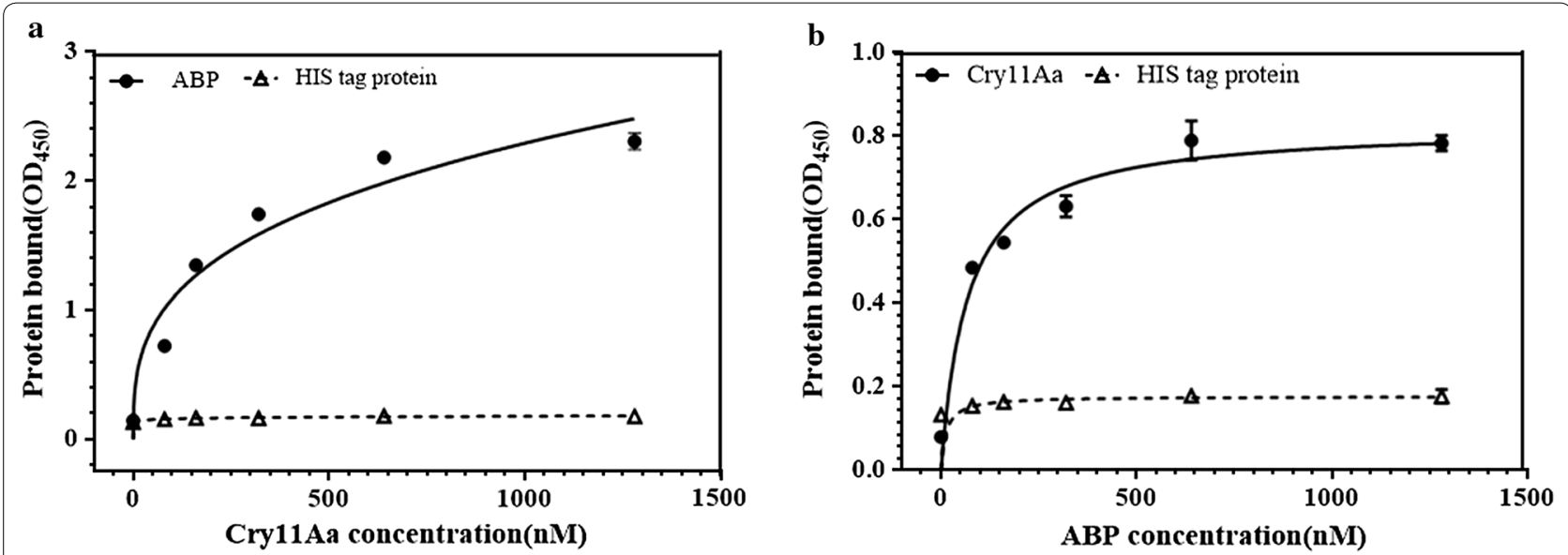

Fig. 7 ELISA analysis between Cry 11 Aa and ATP-binding protein. a Total binding of ABP to Cry $11 \mathrm{Aa}$ and $\mathbf{b}$ total binding of Cry $11 \mathrm{Aa}$ binding to ABP. Each bar represents the mean \pm SD of three technical replicates

\section{Conclusions}

Mosquitoes play an important role in many infectious diseases. ABP is detected in many insects (including mosquitoes) related to the process of the Bti toxin reaction, which is widely used in mosquito biocontrol. In order to clarify the function of ATP-binding protein in Ae. aegypti against Bti toxin, the ABP gene of Ae. aegypti (GenBank: XM_001661856.2) was cloned, expressed and purified. Furthermore, results of farwestern blot and ELISA showed an interaction between ABP and Cry11Aa. Our bioassay also showed that the mortality of Ae. aegypti increased with more the ABP added. Our results suggest that $\mathrm{ABP}$ in Ae. aegypti can 


\section{modulate the toxicity of Cry11Aa toxin to the mosquito by binding to the Bti toxin.}

\begin{abstract}
Abbreviations
ABC transporters: ATP-binding cassette transporters; Bt: Bacillus thuringiensis; Bti: Bacillus thuringiensis israelensis; ALP: alkaline phosphatase; APN: aminopeptidase-N; LB: Luria-Bertani; Trx: thioredoxin; PCR: polymerase chain reaction; SDS-PAGE: sodium dodecyl sulfate polyacrylamide gel electrophoresis; IPTG: isopropyl- $\beta$-D-1-thiogalactopyranoside; ELISA: enzyme-linked immunosorbent assay; PBS: phosphate-buffered saline; PBS-T: phosphate-buffered saline containing Tween-20.
\end{abstract}

\section{Acknowledgements}

All authors gratefully acknowledge Dr Sarjeet Gill, University of California, Riverside for providing the Cry 11 Aa recombinant Bt strain used in this study.

\section{Authors' contributions}

$X Q Y$ and $L L Z$ conceived and designed the experiments. GHZ, JNL, XYJ, XHH, $\mathrm{CXW}, \mathrm{KB}, \mathrm{ZHY}$ and $\mathrm{XHH}$ performed the experiments. GHZ, XHH and $\mathrm{LLZ}$ analyzed the data and wrote the manuscript. JW, HC, EJH, XG, XQY and LLZ revised the paper. All authors read and approved the final manuscript.

\section{Funding}

This work was funded by the National Program of China (Grant Numbers 2017YFE0121700, 2017YFE0122000 and 2017YFD0200400), the National Natural Science Foundation of China (NSFC, Grant Number 31301724), the National Institute of Health (Grant Number Al117808), the Foreign Cooperative Project of Fujian Province (Grant Number 201810023), the Science and Technology Project of Fuzhou (Grant Number 2018-G-70), the Open Project Funds of State Key Laboratory of Pathogen and Biosecurity (SKLPBS1838) and Key Laboratory of Green Control of Insect Pests in Fujian Province (PTJH1700701), and the Special Fund for Scientific and Technological Innovation of Fujian Agriculture and Forestry University (Grant Numbers CXZX2017136, CXZX2017306, CXZX2017214 and KF2015064-65).

\section{Availability of data and materials}

Data supporting the conclusions of this article are included within the article. The raw data are available from the corresponding author upon request. The sequence of the ATP-binding protein used in this paper refers to the NCBI GenBank accession number XM_001661856.2.

\section{Ethics approval and consent to participate}

This study was conducted in accordance with the recommendations set forth in the Animal Ethics Procedures and Guidelines of the People's Republic of China. The experiment rabbits were maintained in an enriched environment of the animal laboratory, Fujian Academy of Medical Sciences, China. All animal experiments were carried out according to the Animal Administration and Ethics Committee of Fujian Agriculture and Forestry University (China). All efforts were made to minimize the number of animals used and their suffering.

\section{Consent for publication}

Not applicable.

\section{Competing interests}

The authors declare that they have no competing interests.

\footnotetext{
Author details

${ }^{1}$ State Key Laboratory of Ecological Pest Control for Fujian and Taiwan Crops \& School of Life Science, Fujian Agriculture and Forestry University, Fuzhou 350002, China. ${ }^{2}$ Fujian International Travel HealthCare Center, Fuzhou 350001, China. ${ }^{3}$ Guangzhou Key Laboratory of Insect Development Regulation and Application Research, Institute of Insect Science and Technology \& School of Life Sciences, South China Normal University, Guangzhou 510631, China. ${ }^{4}$ Division of Cell Biology and Biophysics, University of Missouri-Kansas City, Kansas City, MO 64110, USA.
}

Received: 27 December 2018 Accepted: 9 June 2019

Published online: 25 June 2019

\section{References}

1. Turell MJ, Dohm DJ, Sardelis MR, Oquinn ML, Andreadis TG, Blow JA. An update on the potential of north American mosquitoes (Diptera: Culicidae) to transmit West Nile virus. J Med Entomol. 2005:42:57-63.

2. Zhang LL, Wu SQ, Peng Y, Li MW, Sun LY, Huang EJ, et al. The potential of the novel mosquitocidal Bacillus thuringiensis strain LLP29 for use in practice. J Vector Ecol. 2011;36:458-60.

3. Ben-Dov E. Bacillus thuringiensis susbsp. israelensis and its dipteran-specific toxins. Toxins (Basel). 2014;6:1222-43.

4. Eivazzadeh-Keihan R, Pashazadeh-Panahi P, Mahmoudi T, Chenab KK, Baradaran B, Hashemzaei M, et al. Dengue virus: a review on advances in detection and trends-from conventional methods to novel biosensors. Mikrochim Acta. 2019;186:329.

5. Epelboin Y, Talaga S, Epelboin L, Dusfour I. Zika virus: an updated review of competent or naturally infected mosquitoes. PLoS Negl Trop Dis. 2017;11:e0005933.

6. Zhang Q, Hua G, Adang MJ. Effects and mechanisms of Bacillus thuringiensis crystal toxins for mosquito larvae. Insect Sci. 2017;24:714-29.

7. Zhao L, Alto BW, Duguma D. Transcritional profile for detoxification enzymes AeaGGT1 and AqeGGT2 from Aedes aegypti (Diptera: Culicidae) in response to larvicides. J Med Entomol. 2017;54:878-87.

8. Paiva MH, Lovin DD, Mori A, Melo-Santos MA, Severson DW, Ayres CF. Identification of a major quantitative trait locus determining resistance to the organophosphate temephos in the dengue vector mosquito Aedes aegypti. Genomics. 2016;107:40-8.

9. Likitvivatanavong S, Chen J, Bravo A, Soberón M, Gill SS. Cadherin, alkaline phosphatase, and aminopeptidase $\mathrm{N}$ as receptors of $\mathrm{Cry} 11 \mathrm{Ba}$ toxin from Bacillus thuringiensis subsp. jegathesan in Aedes aegypti. Appl Environ Microbiol. 2011;77:24-31.

10. Stalinski R, Laporte F, Despres L, Tetreau G. Alkaline phosphatases are involved in the response of Aedes aegypti larvae to intoxication with Bacillus thuringiensis subsp. israelensis Cry toxins. Environ Microbiol. 2016;18:1022-36.

11. Schnepf E, Crickmore N, Van Rie J, Lereclus D, Baum J, Feitelson J, et al. Bacillus thuringiensis and its pesticidal crystal proteins. Microbiol Mol Biol Rev. 1998;62:775-806.

12. Vallet-Gely I, Lemaitre B, Boccard F. Bacterial strategies to overcome insect defences. Nat Rev Microbiol. 2008;6:302-13.

13. Berry C, O’Neil S, Ben-Dov E, Jones AF, Murphy L, Quail MA, et al. Complete sequence and organization of pBtoxis, the toxin-coding plasmid of Bacillus thuringiensis subsp. israelensis. Appl Environ Microb. 2002;68:5082-95

14. Zhang LL, Huang EJ, Tang BZ, Guan X, Gelbic I. Mosquito cell line C6/36 shows resistance to Cyt1Aa6. Indian J Exp Biol. 2012;50:265-9.

15. Ben-Dov E. Bacillus thuringiensis subsp. israelensis and its dipteran-specific toxins. Toxins. 2014;6:1222-43.

16. Bravo A, Gill SS, Soberon M. Mode of action of Bacillus thuringiensis Cry and Cyt toxins and their potential for insect control. Toxicon. 2007;49:423-35.

17. Gomez I, Pardo-Lopez L, Munoz-Garay C, Fernandez LE, Perez C, Sanchez $J$, et al. Role of receptor interaction in the mode of action of insecticidal Cry and Cyt toxins produced by Bacillus thuringiensis. Peptides. 2007;28:169-73.

18. Palma L, Muñoz D, Berry C, Murillo J, Caballero P. Bacillus thuringiensis toxins: an overview of their biocidal activity. Toxins. 2014;6:3296-325.

19. Lu KY, Gu YQ, Liu XP, Lin Y, Yu XQ. Possible insecticidal mechanisms mediated by immune response related Cry-binding proteins in the midgut juice of Plutella xylostella and Spodoptera exigua. J Agric Food Chem. 2017;65:2048-55.

20. Ideo H, Fukushima K, Gengyo-Ando K, Mitani S, Dejima K, Nomura K, et al. A Caenorhabditis elegans glycolipid-binding galectin functions in host defense against bacterial infection. Biol Chem. 2009;284:26493-501.

21. Zhang LL, Hu XH, Wu SQ, Batool K, Chowdhury M, Lin Y, et al. Aedes aegypti galectin competes with Cry11Aa for binding to ALP1 to modulate Cry toxicity. J Agric Food Chem. 2018;66:13435-43. 
22. Yassine HA. Functional interactions between C-type lectins and clipdomain serine protease homologs in A. gambiae immune responses. Ph.D. Thesis, American University of Beirut; 2013.

23. Batool K, Alam I, Zhao GH, Wang JX, Xu J, Yu XQ, et al. C-type lectin-20 interacts with ALP1 receptor to reduce Cry toxicity in Aedes aegypti. Toxin. 2018;10:390.

24. Gahan $L$, Pauchet $Y$, Vogel $H$, Heckel DG. An ABC transporter mutation is correlated with insect resistance to Bacillus thuringiensis Cry1Ac toxin. PLoS Genet. 2010;6:e1001248.

25. Liu CX, Xiao YT, Li XC, Oppert B, Tabashnik BE, Wu KM. Cis-mediated down-regulation of a trypsin gene associated with Bt resistance in cotton bollworm. Sci Rep. 2014;4:7219.

26. Park Y, Gonzalez-Martinez RM, Navarro-Cerrillo G, Chakroun M, Kim Y, Ziarsolo $P$, et al. ABCC transporters mediate insect resistance to multiple Bt toxins revealed by bulk segregant analysis. BMC Biol. 2014;12:46.

27. Wu Y. Detection and mechanisms of resistance evolved in insects to Cry toxins from Bacillus thuringiensis. Adv Insect Physiol. 2014;47:297-342.

28. Qi WP, Ma XL, He WY, Chen W, Zou MM, Gurr GM, et al. Characterization and expression profiling of ATP-binding cassette transporter genes in the diamondback moth, Plutella xylostella (L.). BMC Genom. 2016;17:760.

29. Atsumi S, Miyamoto K, Yamamoto K, Narukawa J, Kawai S, Sezutus H, et al. Single amino acid mutation in an ATP-binding cassette transporter gene causes resistance to Bt toxin Cry1 Ab in the silkworm, Bombyx mori. Proc Natl Acad Sci USA. 2012;109:E1591-8.

30. Tanaka S, Miyamoto K, Noda H, Jurat-Fuentes JL, Yoshizawa Y, Endo H, et al. The ATP-binding cassette transporter subfamily $C$ member 2 in Bombyx mori larvae is a functional receptor for Cry toxins from Bacillus thuringiensis. FEBS J. 2013;280:1782-94.

31. Zhang LL, Zhao GH, Hu XH, Liu JN, Li MW, Batool K, et al. Cry11Aa interacts with the ATP-binding protein from Culex quinquefasciatus to improve the toxicity. J Agric Food Chem. 2017:65:10884-90.
32. Zhang LL, Huang EJ, Lin J, Gelbic I, Zhang QL, Guan Y, et al. A novel mosquitocidal Bacillus thuringiensis strain LLP29 isolated from the phylloplane of Magnolia denudata. Microbiol Res. 2010;165:133-41.

33. Chen JW, Aimanova K, Gill SS. Functional characterization of Aedes aegypti alkaline phosphatase ALP1 involved in the toxicity of Cry toxins from Bacillus thuringiensis subsp. israelensis and jegathesan. Peptide. 2017;98:78-85

34. Chen JW, Aimanova KG, Pan S, Gill SS. Identification and characterization of Aedes aegypti aminopeptidase $\mathrm{N}$ as a putative receptor of Bacillus thuringiensis Cry1 1A toxin. Insect Biochem Mol. 2009;39:688-96.

35. Chen JW, Likitvivatanavong S, Aimanova KG, Gill SS. A 104 kDa Aedes aegypti aminopeptidase $\mathrm{N}$ is a putative receptor for the Cry11Aa toxin from Bacillus thuringiensis subsp. israelensis. Insect Biochem Mol Biol. 2013:43:1201-8.

36. World Health Organization. WHO specifications and evaluations for public health pesticides Bacillus thuringiensis subspecies israelensis strain AM65-52. Geneva: World Health Organization; 2007.

37. Guo Z, Kang S, Zhu X, Xia J, Wu Q. Down-regulation of a novel ABC transporter gene (Pxwhite) is associated with Cry1 Ac resistance in the diamondback moth, Plutella xylostella, (L.). Insect Biochem Mol Biol. 2015;59:30-40

38. Zhou Z, Wang Z, Liu Y, Liang G, Shu C, Song F, et al. Identification of $A B C C 2$ as a binding protein of Cry1Ac on brush border membrane vesicles from Helicoverpa armigera by an improved pull-down assay. Microbiologyopen. 2016;5:659-69.

\section{Publisher's Note}

Springer Nature remains neutral with regard to jurisdictional claims in published maps and institutional affiliations.
Ready to submit your research? Choose BMC and benefit from:

- fast, convenient online submission

- thorough peer review by experienced researchers in your field

- rapid publication on acceptance

- support for research data, including large and complex data types

- gold Open Access which fosters wider collaboration and increased citations

- maximum visibility for your research: over $100 \mathrm{M}$ website views per year

At BMC, research is always in progress.

Learn more biomedcentral.com/submissions 